\title{
Complex response of a bistable laminated plate: Multiscale entropy analysis
}

\author{
M. Borowiec ${ }^{1}$, A. Rysak ${ }^{1}$, D.N. Betts ${ }^{2}$, C.R. Bowen ${ }^{2}$, H.A. Kim², and G. Litak ${ }^{1, a}$ \\ ${ }^{1}$ Faculty of Mechanical Engineering, Lublin University of Technology, Nadbystrzycka 36, 20-807 Lublin, Poland \\ 2 Department of Mechanical Engineering, University of Bath, Bath, BA2 7AY, UK
}

Received: 14 April 2014 / Revised: 3 July 2014

Published online: 6 October 2014

(C) The Author(s) 2014. This article is published with open access at Springerlink.com

\begin{abstract}
The use of bistable laminates is a potential approach to realize more broadband piezoelectric based energy harvesting systems. Based on the experimental time series of a bistable laminate plate we have examined its dynamic response. The system was subjected to harmonic excitations showing the existence of single well and snap-through vibrations of periodic and chaotic character. To identify the dynamics of the system response we examine the frequency spectrum, phase portraits and multi-scaled entropy. It is observed that the composite multiscale entropy analysis can be used to identify complexity within the dynamic response successfully.
\end{abstract}

\section{Introduction}

With the recent increase in the use of wireless sensor networks and electronics requiring a portable and autonomous energy source, energy harvesting devices have been developed in an attempt to convert ambient vibrations to electrical energy via electrostatic generation, electromagnetic induction, and the piezoelectric effect [1,2]. The general need is to harvest electrical energy from waste mechanical energy in the form of ambient vibrations, which generates an alternating voltage using a piezoelectric material. These materials are of interest due to their higher strain energy densities compared to electrostatic and electromagnetic systems, and the simplicity and solid-state nature of the strain energy to electrical energy conversion [3]. For practical applications the alternating voltage is converted to a stable rectified voltage through an AC-DC converter and is stored in a battery or capacitor so that it can be subsequently used to power a low-power device, such as a wireless sensor network for structural health monitoring.

In some cases the mechanical vibration to be harvested is of a specific frequency, e.g. a motor rotating at a fixed speed, and a resonant based harvesting system in appropriate ambient conditions. However, in many cases the vibration consists of a range of frequencies, such as those on railway carriages [4] or other forms of transport [5,6]. It has been reported that introducing a nonlinear stiffness can lead to an improvement of the frequency bandwidth of the harvester [7]. As a result, a variety of approaches for incorporating nonlinearity in the stiffness of energy harvesters have been considered, most notably by designing bistable harvesters with two distinct energy wells [8-11]. One recent approach is to utilise the structural bistability of asymmetric composite laminates. This was initially explored by Arrieta et al. [12] who employed a piezoelectric element attached to the surface of a bistable laminate with an asymmetric stacking sequence, exploiting the inherent bistability which can be designed in this specific family of laminated plates [13]. For energy harvesting [14] when a flexible piezoelectric material is attached to the bistable laminate surface it can generate power by repeated straining as it experiences snap-through from each stable state as a result of mechanical vibrations. Experimentally, such harvesting devices have been shown to exhibit high levels of power extraction over a wide range of frequencies when harmonically excited from a central mounting [15], with scope for improved power generation through changes in the geometry highlighted.

In the present work we use an electro-mechanical system to generate mechanical vibrations leading to snap-through of the laminate between its two stable states. Such system can demonstrate a frequency broad-band effect of voltage output. The present motivation is the ability to identify the bistable, mechanical resonator response such as single well oscillations, continuous snap-through between stable states or chaotic or periodic snap-through behavior [15]. This information could be used to optimize the ambient energy harvesting.

\footnotetext{
${ }^{a}$ e-mail: g.litak@pollub.pl
} 


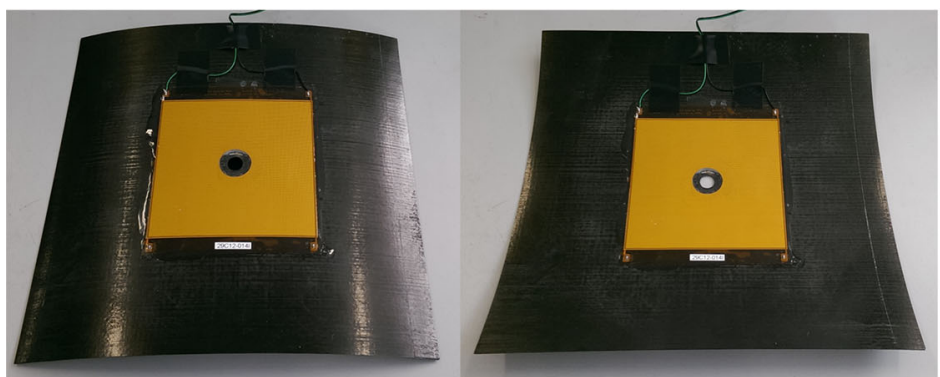

(a)

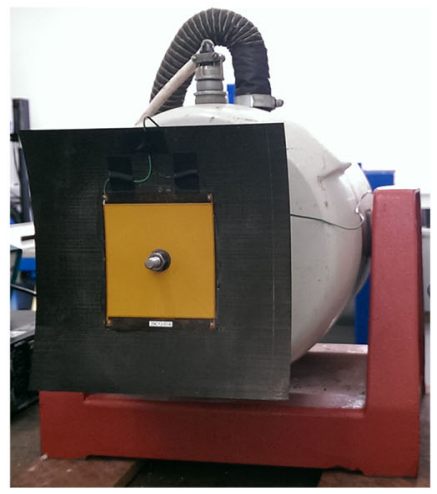

(b)

Fig. 1. (a) Stable shapes of a $190 \mathrm{~mm} \times 190 \mathrm{~mm}[0 / 90]_{T}$ bistable laminate plate, and (b) experimental setup showing mechanical shaker attachment. A square CFPR laminate is coupled to smaller square piezoelement (MFC, Micro-Fibre-Composite) but the output voltage is not measured in this experiment.

Table 1. Statistics of the experimental results (figs. 2(a)-(c)).

\begin{tabular}{|l|c|c|c|c|}
\hline Case & $\begin{array}{c}\text { Excitation frequency } \\
{[\mathrm{Hz}]}\end{array}$ & $\begin{array}{c}\text { Input of acceleration } \\
{[\mathrm{g}]}\end{array}$ & $\begin{array}{c}\text { St. dev. of the corner displacement } \\
{[\mathrm{mm}]}\end{array}$ & $\begin{array}{c}\text { St. dev. of the corner velocity } \\
{[\mathrm{m} / \mathrm{s}]}\end{array}$ \\
\hline (a) & 58 & 3 & 1.53 & 0.68 \\
(b) & 55 & 5.5 & 4.25 & 1.07 \\
(c) & 55 & 5.7 & 4.11 & 1.04 \\
\hline
\end{tabular}

\section{Experimental setup and results}

The experimental system is presented in fig. 1. A square $[0 / 90]_{T}$ carbon fibre reinforced laminate is considered as the basis for developing a broadband energy harvesting device. The laminate measures $190 \mathrm{~mm}$ by $190 \mathrm{~mm}$ and is made from M21/T800 CFRP prepreg material. A single piezoelectric Macro Fiber Composite (MFC) layer (M8585$\mathrm{P} 2,85 \mathrm{~mm} \times 85 \mathrm{~mm}$ ) is bonded to the laminate surface. The laminate is mounted to the electrodynamic shaker (LDS V455) at its centre. Under kinematic excitation the laminate plate can show different responses which are recordered by laser vibrometer (Polytec PSV-400-M4 with VD-09 decoder) at one of the plate corners.

The time series of three characteristic responses are plotted in fig. 2; the experimental conditions are in table 1. It is possible to observe a single well mode case (fig. 2(a)) with small displacements, and snap-through with larger displacements in regular (fig. 2(b)) and chaotic (fig. 2(c)) cases. The additional points show reduced sampling appropriate for calculation of the Multiscaled Entropy in the following section. The corresponding Fourier spectra for these three conditions are presented in figs. 3(a)-(c). Note that the cases in figs. 3(a) and (b) are localized in frequencies is expected for periodic vibrations, while fig. 3(c) shows a continuously smeared spectrum that is typical for chaotic behaviour. Note that cases in figs. 3(b) and (c) are similar, as the periodic case is characterized by a large number of peaks appearing in selected multiple frequencies. As expected for any experimental data, the time series is also influenced by noise. However it is possible to detect single, albeit fairly broader lines, in the phase portraits in fig. 4 . Interestingly, in fig. 4(b) one can observe a multiple loop structure (based on smeared lines) which is consistent with the multi frequency response (fig. 3(b)). Note that this pattern of lines is destroyed for the chaotic response (fig. 3(c)).

\section{Composite multiscale entropy analysis}

To improve understanding of the behaviour of complex systems that manifest themselves in nonlinear behaviour, sample entropy analysis is becoming increasingly more popular [16-22]. This method provides, for measured signals, a relative level of complexity of finite length time series. Unfortunately, there is no consensus of the complexity definition, but it is combined with "meaningful structural richness" [23] contained over multiple spatio-temporal correlations.

The concept of multi-scale entropy (MSE) [18-22] is based on the coarse-graining procedure that uses a coarsegrained time series, as an average of the original data points within nonoverlapping windows by increasing the scale factor $\tau$ according to formula (eq. (1)):

$$
y_{j}^{(\tau)}=\frac{1}{\tau} \sum_{i=(j-1) \tau+1}^{j \tau} x_{i}
$$



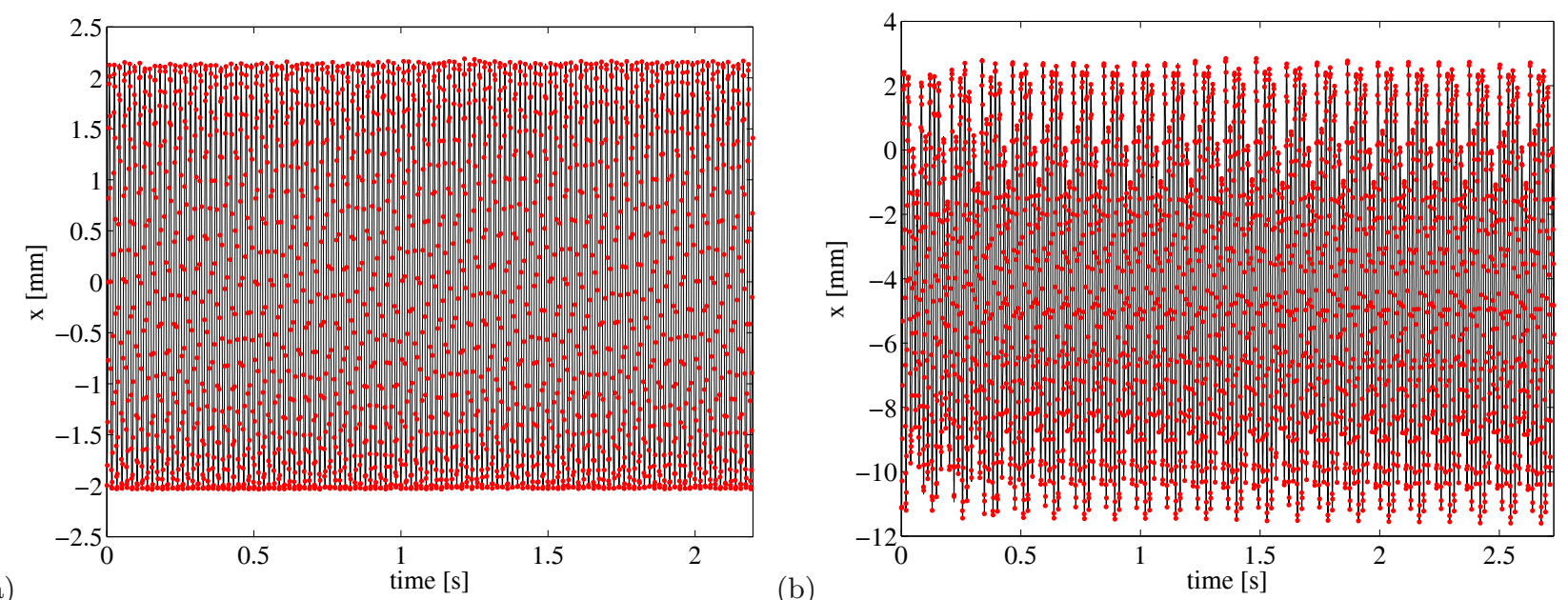

(b)

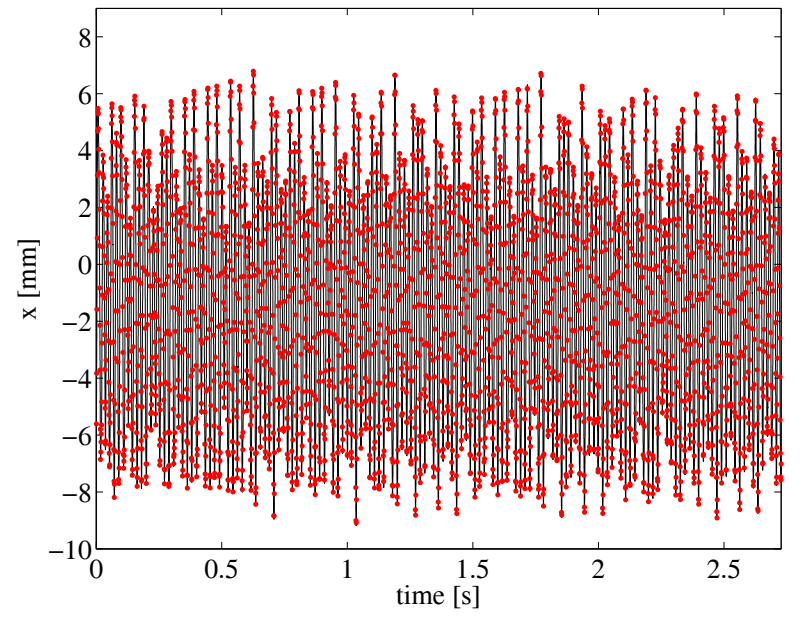

Fig. 2. Time series of experimental results, for a single corner point mass, corresponding to single well mode case (a), and snapthrough buckling: regular (b), chaotic (c) cases. The red points plotted along the time series show reduced sampling appropriate for calculation of the multiscaled entropy.

where $x$ is a raw one-dimensional time series $x=\left\{x_{1}, x_{2}, \ldots, x_{N}\right\}$. In this approach for each scale factor $\tau$, the MSE calculation based on the time series of the coarse-grained $y_{j}^{(\tau)}$ :

$$
\operatorname{MSE}(\mathbf{x}, \tau, m, r)=\operatorname{SampEn}\left(\mathbf{y}_{1}^{(\tau)}, m, r\right) .
$$

where $m=2$ is the pattern length and $r$ is the similarity criterion and is usually chosen to be $r<\sigma[20]$, here $\sigma$ is the standard deviation of the original time series.

To estimate $\operatorname{SampEn}\left(\mathbf{y}_{1}^{(\tau)}, m, r\right)$ from eq. (2) (see also fig. 5) we count the number of vector pairs denoted by $y_{1}(i)$ and $y_{1}(j)$ in the time series of length $m$ and $m+1$ having distance $d\left[y_{1}(i), y_{1}(j)\right]<r$. We denote them by $N_{m}$ and $N_{m+1}$, respectively. Finally, we define the sample entropy to be [17]

$$
\operatorname{SampEn}\left(\mathbf{y}_{1}^{(\tau)}, m, r\right)=-\log \frac{N_{m+1}}{N_{m}} .
$$

Strictly speaking, it is the minus of the logarithm of the conditional probability that two sequences with a tolerance $r$ form points that remain within $r$ of each other at the next point.

Continuing these research efforts Wu et al. [24] introduced the concept of a composite multi-scale entropy (CMSE), which for higher scale factor provides entropy more reliably than the usual multi-scale entropy by including multiple combinations of neighbour points. The prescribed algorithm for CMSE calculations is formula (eq. (4)):

$$
\operatorname{CMSE}(\mathbf{x}, \tau, m, r)=\frac{1}{\tau} \sum_{k=1}^{\tau} \operatorname{SampEn}\left(\mathbf{y}_{k}^{(\tau)}, m, r\right) .
$$




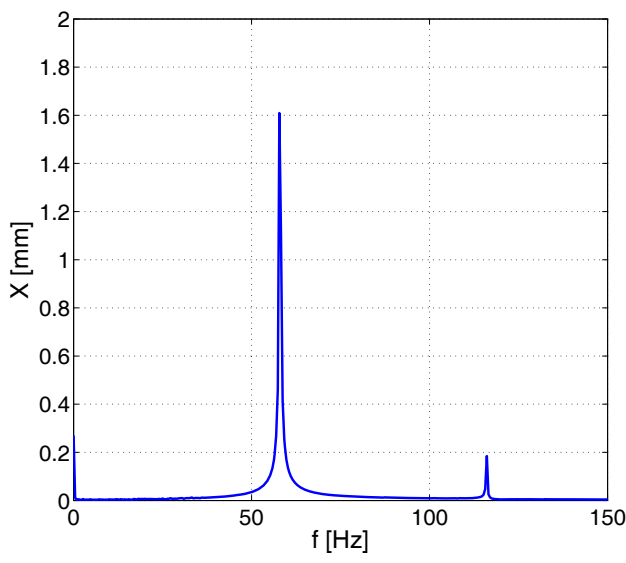

(a)

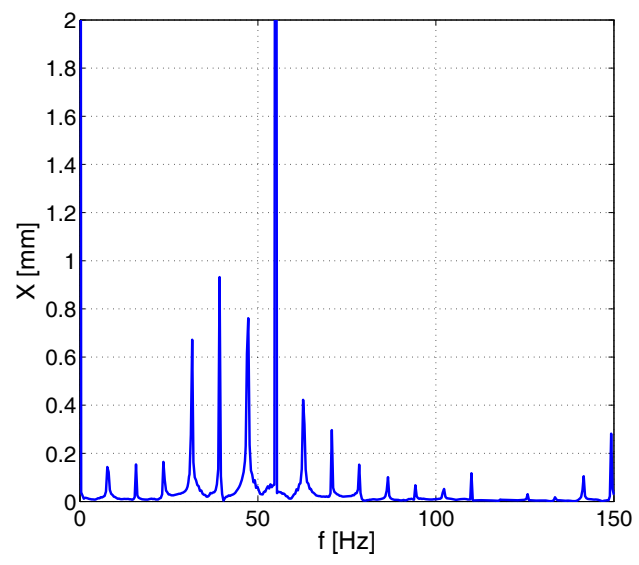

(b)

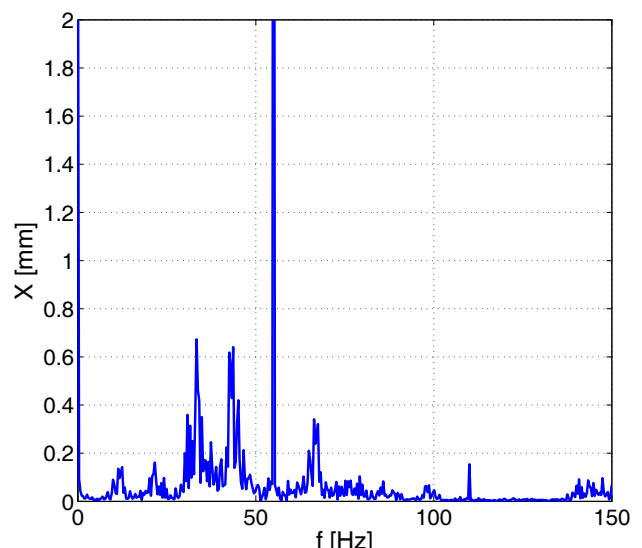

(c)

$\mathrm{f}[\mathrm{Hz}]$

Fig. 3. Fourier spectra of the full measured time series for three different cases. The cases in panels (a)-(c) correspond to fig. $2(\mathrm{a})-(\mathrm{c})$.

The algorithm of the composite multi-scale entropy is presented in fig. 5. In the present paper the CMSE is applied for real signals reporting a different behaviour of the system characterized by the time series in fig. 2 . The results of composite multi-scale entropy calculation are provided for several similarities $r$ for different CMSE values with increasing scale factor $\tau$. The existence of higher values of CMSE corresponds to the existence of more complexity within the analysed signal. It can be noticed that there is a variation in the irregularity of the system as a different $\tau$ is chosen. These results are able to confirm the validity of others analysis reported in the paper [25] by means of the recurrence plots at decomposed signal modes.

The results of the CMSE for $m=2$ are presented in fig. 6. Note that the smallest value of CMSE and also its smallest oscillations are reached for the largest similarity factor $r$. On examination of figs. 6(a) and (b) we observe that in various regions of scale factor, small and large limits of similarity factor $r$ may cause the CMSE to occasionally approach to zero. For relatively large $r\left(r=\sigma_{x}\right.$ - blue line) this tendency is governed by the period of oscillations that account for strong averaging. Consequently, the effective (coarse-grained) sequence is averaged out for the certain scale factor, $\tau \gtrsim 15$. The regular-periodic solutions (figs. 6(a) and (b)) show larger fluctuations of CMSE with frequent decreases to small values. Interestingly, reaching fairly small, almost nodal value is realized for $r=0.01 \sigma_{x}$. This is a signature that the system is characterised by specific periods (see also figs. 3(a) and (b)). On the other hand, in the chaotic (fig. 6(c)) case, we observe a monotonic substantial increase of the CMSE values with decreasing $r$ which is typical for a chaotic response [26]. The slightly decreasing trend of CMSE against the scale factor $r$ indicates long range correlated noise [18] which could be interpreted as an effect of chaotic behaviour. This is the principal result of our investigations. Clearly, the CMSE approach enables to distinguish the chaotic solution using the criterion of the finite entropy value in the limit of small similarity factor $r$. The ability to detect the onset of snap-through of the bistable laminate in a chaotic or continuous (repeatable) manner is of importance to understand the complex dynamics of nonlinear harvesting systems and ultimately generate maximum power output for a given broadband ambient vibration. 

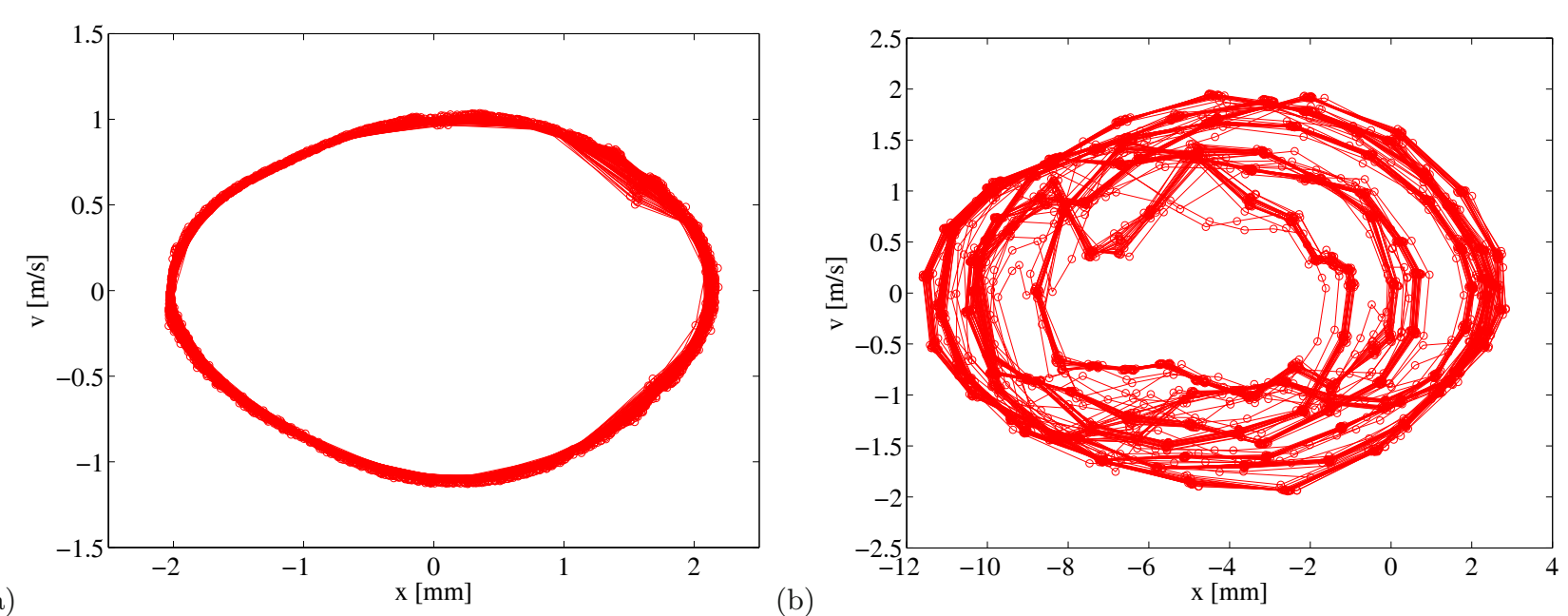

(a)

(b)

(c)

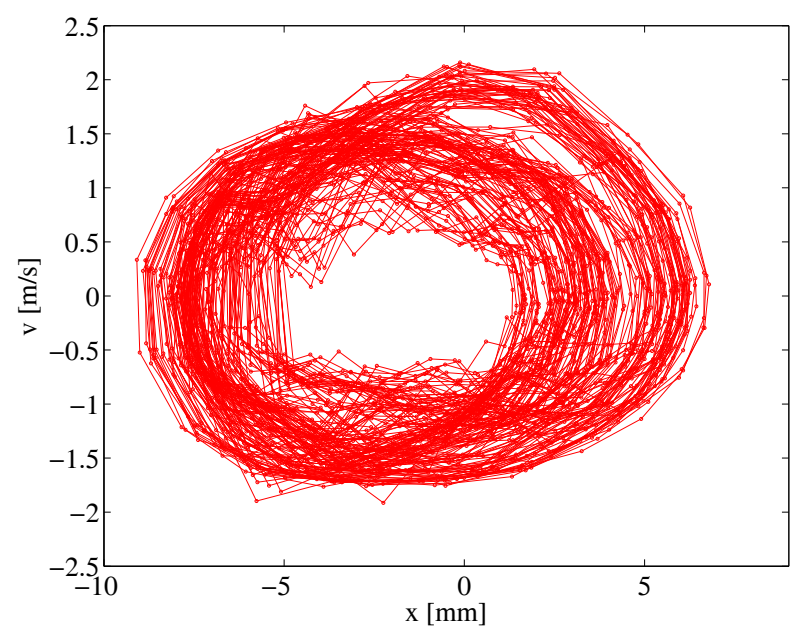

Fig. 4. Phase portraits based on the reduced sampling corresponding to single well mode case (a), and snap-through: regular (b), chaotic (c) cases.

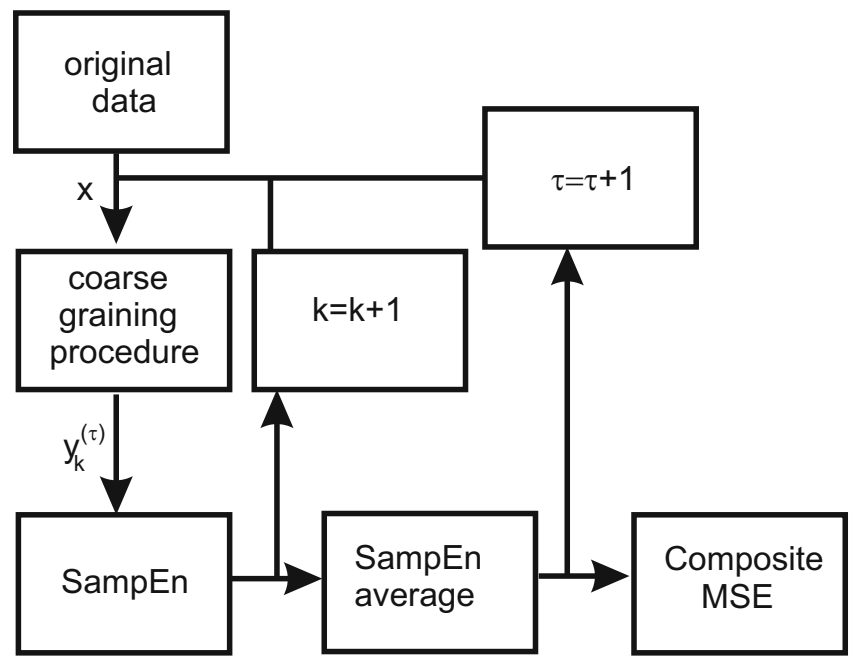

Fig. 5. The algorithm of composite multi-scale entropy. 
(a)

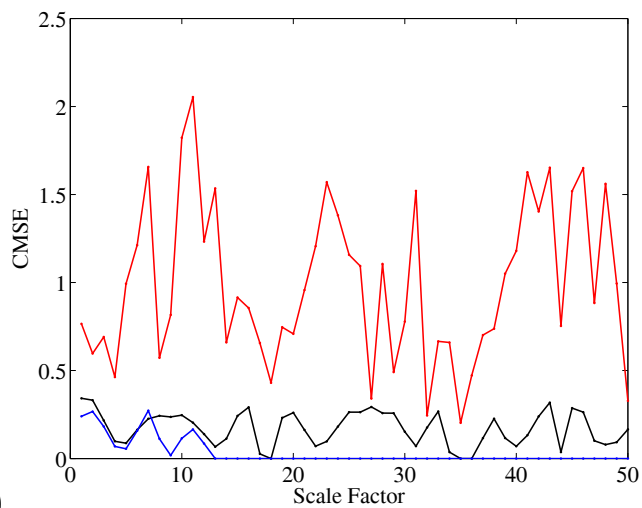

(b)

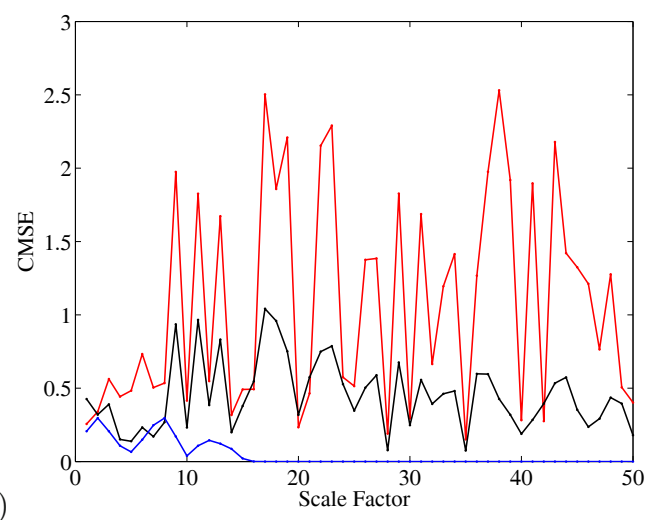

(c)

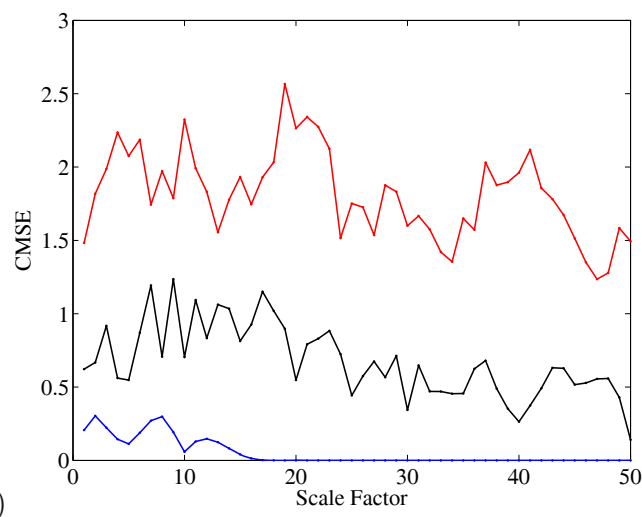

Fig. 6. Composite multiscale entropy CMSE results of the corner point displacement corresponding to small single well oscillations (a), repeatable snap-through (b) and chaotic snap-through (c) cases for illustrated varied similarity factor $r$. Starting from up to down curves: $r=0.01 \sigma_{x}, 0.1 \sigma_{x}, \sigma_{x}$, respectively.

\section{Conclusions}

The use of bistable laminates is considered for piezoelectric based energy harvesting systems. Based on the experimental time series of a bistable laminate plate we have examined its dynamic response. The examined system response is shown to include different solutions, with the potential to be exploited in the harvesting device. To increase the efficiency of the device one has to identify the wave dynamics along the plate and in the region of piezoelectric transducer. We demonstrated that the multiscale entropy can be helpful in the response characterization. Using the simple approach of a single point laser vibrometer measurement we note that the multiscale nature of the phenomenon is reflected in the calculated entropy and this method (CMSE) could be used to identify the desired dynamic solution. Practically, such a signal analysis approach can be used to identify dynamic modes and optimize an energy harvesting device in the appropriate switching mechanism between solutions. Note that a single point laser measurement simplifies the identification procedure. However, for better understanding of snap-through phenomena dynamics and response identification we are planing perform more systematic experiments by a scanning vibrometer and apply the CMSE concept to multi-point time series, by employing, e.g., the local maximum-entropy approximation schemes [27,28].

The authors gratefully acknowledge the support of the Polish National Science Center (MB and AR) under Grant No. 2013/11/D/ST8/03308. Bowen acknowledges funding from the European Research Council under the European Union's Seventh Framework Programme (FP/2007-2013) / ERC Grant Agreement no. 320963 on Novel Energy Materials, Engineering Science and Integrated Systems (NEMESIS). Kim acknowledges financial support from the Engineering and Physical Science Research Council (EPSRC) for Project Reference: EP/J014389/1 "Optimisation of Broadband Energy Harvesters Using Bistable Composites".

Open Access This is an open access article distributed under the terms of the Creative Commons Attribution License (http://creativecommons.org/licenses/by/4.0), which permits unrestricted use, distribution, and reproduction in any medium, provided the original work is properly cited. 


\section{References}

1. H.A. Sodano, D.J. Inman, G. Park, Shock Vibration Digest 36, 197 (2004).

2. C.R. Bowen, H.A. Kim, P.M. Weaver, S. Dunn, Energy Environ. Sci. 7, 25 (2014).

3. S. Priya, J. Electroceram. 19, 167 (2007).

4. S.H. Ju, H.T. Lin, J.Y. Huang, J. Sound Vib. 319, 247 (2009).

5. Q. Zhu, M. Guan, Y. He, in International Conference on Information and Automation Conference Proceedings, Shenyang, China, 6-8 June 2012 (IEEE, 2012) pp. 349-354.

6. O.J. Wood, C.A. Featherston, D. Kennedy, M. Eaton, R. Pullin, Key Eng. Mat. 518, 246 (2012).

7. A. Erturk, J. Hoffmann, D.J. Inman, Appl. Phys. Lett. 94, 1 (2009).

8. S.C. Stanton, C.C. McGehee, B.P. Mann, Appl. Phys. Lett. 95, 174103 (2009).

9. S.C. Stanton, C.C. McGehee, B.P. Mann, Physica D 239, 640 (2010).

10. A. Erturk, D.J. Inman, J. Sound Vib. 330, 2339 (2011).

11. R.L. Harne, M. Thota, K.W. Wang, Appl. Phys. Lett. 102, 053903 (2013).

12. A.F. Arrieta, P. Hagedorn, A. Erturk, D.J. Inman, Appl. Phys. Lett. 97, 104102 (2010).

13. M.W. Hyer, J. Compos. Mater. 15, 175 (1981).

14. D.N. Betts, H.A. Kim, C.R. Bowen, D.J. Inman, Appl. Phys. Lett. 100, 114104 (2012).

15. D.N. Betts, R.A. Guyer, P.-Y. Le Bas, C.R. Bowen, D.J. Inman, H.A. Kim, in Proceedings of "AIAA SciTech Conference", Maryland, USA (2014).

16. S.M. Pincus, Proc. Natl. Acad. Sci. U.S.A. 88, 2297 (1991).

17. J.S. Richman, J.R. Moorman, Am. J. Physiol. Heart Circ. Physiol. 278, H2039 (2000).

18. A.L. Goldberger, L.A.N. Amaral, L. Glass, J.M. Hausdorff, P.Ch. Ivanov, R.G. Mark, J.E. Mietus, G.B. Moody, C.-K. Peng, H.E. Stanley, Circulation 101, E215 (2000) Circulation Electronic Pages, http://circ.ahajournals.org/cgi/content/ full/101/23/e215 2000 (June 13).

19. M. Costa, A.L. Goldberger, C.-K. Peng, Phys. Rev. Lett. 89, 062102 (2002).

20. M. Costa, C.-K. Peng, A.L. Goldberger, J.M.. Hausdorff, Physica A 330, 53 (2003).

21. M. Costa, A.L. Goldberger, C.-K. Peng, Phys. Rev. E 71, 021906 (2005).

22. M. Borowiec, A.K. Sen, G. Litak, J. Hunicz, G. Koszalka, A. Niewczas, Forschung. Ing. 74, 99 (2010).

23. P. Grassberger, Information and complexity measures in dynamical systems, in Information Dynamics, edited by H. Atmanspacher, H. Scheingraber (Plenum Press, 1991) pp. 15-33.

24. S.-D. Wu, C.-W. Wu, S.-G. Lin, C.-C. Wang, K.-Y. Lee, Entropy 15, 1069 (2013).

25. G. Litak, R. Rusinek, K. Kecik, A. Rysak, A. Syta, in Discontinuity and Complexity in Nonlinear Physical Systems, edited by J.T.A. Machado, D. Baleanu, A. Luo, Vol. 6 (Springer, 2014) p. 359.

26. G. Litak, S. Schubert, G. Radons, Nonlinear Dyn. 69, 1255 (2012).

27. M. Arrojo, M. Ortiz, Int. J. Numer. Methods Eng. 65, 2167 (2006).

28. F. Fraternali, C. Lorenz, G. Marcelli, J. Comput. Phys. 231, 528 (2012). 